Modern Physics Letters B

(C) World Scientific Publishing Company

\title{
ON A POSSIBILITY TO MEASURE THERMOELECTRIC POWER IN SNS STRUCTURES
}

\author{
B.J. AHMEDOV \\ Institute of Nuclear Physics and Ulugh Beg Astronomical Institute \\ Ulughbek, Tashkent 100214, Uzbekistan \\ ahmedov@astrin.uzsci.net
}

\begin{abstract}
Two dissimilar Josephson junctions, which are connected to a heater can act as precise batteries. Because of the difference in thermoelectric power of these batteries, circuit with two dissimilar batteries, under heat flow $\Delta T \sim 10^{-5} \mathrm{~K}$ would have a net EMF $10^{-11} \mathrm{~V}$ around the zero-resistance loop leading to a loop's magnetic flux oscillating in time. It is shown its theoretical value is proportional to both the temperature difference as well as the disparity in the thermoelectric powers of the two junctions.
\end{abstract}

PACS Number(s).: 74.50+r, 42.50.Dv

Thermoelectric effects in superconductor - normal metal - superconductor $(S N S)$ junctions have been studied theoretically and observed experimentally in the papers ${ }^{1-5}$. The purpose of this short Letter is to suggest from theoretical considerations a new proposal to measure thermoelectric effects in the SNS structures.

We shall first consider the behaviour of a single Josephson junction when temperatures of its electrodes $S$ have difference $\Delta T$ or, in other words, when there is a heat flow through the junction. We use resistively shunted model of the Josephson junction in which the latter is considered as a circuit made up of the superconducting junction itself and a normal resistance connected in parallel. Then, due to the Kirchoff's first law, $\vec{\jmath}_{s}=\vec{\jmath}_{n}$.

According to Ohm's law a normal component of a current in the junction is $\vec{\jmath}_{n}=\lambda \vec{E}-\lambda \operatorname{grad} \mu_{e}+\lambda \beta \operatorname{grad} T$, where $\lambda$ is electrical conductivity, $\mu_{e}$ is the chemical potential per unit charge and $\beta$ is thermoelectric power.

Density of the superconducting current flowing through the junction is related to the phase difference $\phi=\Delta \vartheta$ of superconducting wavefunction $\psi=n_{s}^{1 / 2} e^{i \vartheta}$ across the junction by ${ }^{6} \vec{\jmath}_{s}=\vec{\jmath}_{c} \sin \phi$, where $\vec{\jmath}_{c}$ is the critical value of electric current density, $n_{s}$ and $m_{s}$ are the density and mass of Cooper pairs.

Using the considered formulae and Josephson equation ${ }^{6}$

$$
-\hbar \frac{\partial \phi}{c \partial t}=\frac{2 e}{c} \mu_{e}-\frac{2 e}{c} \varphi
$$


one can obtain

$$
\vec{\jmath}_{c} \sin \phi=\lambda \operatorname{grad}\left(\frac{\hbar}{2 e} \frac{\partial \phi}{\partial t}\right)+\lambda \beta \operatorname{grad} T
$$

which after integration on $\vec{n} d S$ will give

$$
R I_{c} \sin \phi=\frac{\hbar}{2 e} \frac{\partial \phi}{\partial t}+\beta \Delta T
$$

Here $R=\int d l / \lambda d S$ is the resistance of the normal layer with length $d l, \varphi$ is the scalar potential and $\vec{A}$ is the vector-potential of electromagnetic field, $I_{c}=\int \overrightarrow{\jmath_{c}} \vec{n} d S$ is electric current, $\vec{n}$ is normal vector to the cross section of wire $d S$.

Therefore if thermoelectric current exceeds the critical current of the Josephson junction, then as a consequence of (2), an alternating current (ac) of frequency

$$
\omega=\frac{2 e}{\hbar} \beta \Delta T
$$

is produced. Formula (3) describes thermoelectric ac Josephson effect according to which a temperature difference $\Delta T$ across the $S N S$ junction results in the Josephson oscillation with the frequency $\omega$, which has been predicted in ${ }^{2}$ and experimentally confirmed ${ }^{3}$.

Consider now two dissimilar $S N S$ junctions $I$ and $I I$, which are connected in opposition by superconducting wires to a common heater source and form a loop with inductance $L$. Josephson junctions have unequal thermoelectric powers $\beta_{I}$ and $\beta_{I I}$, respectively, since they are made from the different materials. According to twofluid model two currents flow in the superconductor: the superconducting current of density $\vec{\jmath}_{s}$ and the normal current of density $\vec{\jmath}_{n}$. In the presence of heat flow, the total current density in the interior of a bulk isotropic homogeneous superconductor must be zero (see, for review ${ }^{7-10}$ ) $\vec{\jmath}=\vec{\jmath}_{s}+\vec{\jmath}_{n}=0$ and so

$$
\vec{\jmath}_{n}=\lambda \beta \operatorname{grad} T=-\vec{\jmath}_{s}=\frac{2 n_{s} e}{m_{s}}\left[\hbar g r a d \vartheta-\frac{2 e}{c} \vec{A}\right] .
$$

Now we integrate equation (4) over the contour $l$ which passes through the interior of superconducting ring with two Josephson contacts and use that $\oint \vec{A} d \vec{l}=$ $\int \vec{B} d \vec{S}=\Phi_{b}$ and $\oint \operatorname{grad} \vartheta d \vec{l}=2 \pi n$, where $n$ is integer. Then

$$
\Phi_{b}=n \Phi_{0}+\frac{\hbar c}{2 e}\left(\phi_{I I}-\phi_{I}\right)
$$

where $\phi_{I I}$ and $\phi_{I}$ are the contributions due to the phase discontinuities $(\phi=\Delta \vartheta)$ at the Josephson junctions $I I$ and $I, \Phi_{0}=\pi \hbar c / e=2 \times 10^{-7} \mathrm{Gauss} \cdot \mathrm{cm}^{2}$ is quantum of the magnetic flux.

The rate of change of magnetic flux is related to $\Delta \omega \equiv \omega_{I I}-\omega_{I}$ by

$$
\frac{d \Phi_{b}}{d t}=\frac{d n}{d t} \Phi_{0}+\frac{\hbar c}{2 e} \Delta \omega
$$

so if $\Delta \omega$ is not equal to zero, a magnetic flux $\Delta \Phi_{b} \neq 0$ will be induced. As long as $\Delta \Phi_{b}<\Phi_{0}, n$ will remain constant and $\Delta \Phi_{b}$ will increase linearly with time until $\Delta \Phi_{b}=\Phi_{0}$, then the order of the step $n$ will change as flux quantum enters the loop. 
Thus due to the effect of gradient of temperature on the junctions with unequal thermoelectric powers is equivalent to having a time dependent flux, as given by the last term on the left hand side of equation (6). Using equations (3) and (6) one can derive that the change in magnetic flux $\Phi_{b}$ inside the circuit during the time interval $[0, t]$ subjects to formula

$$
\Delta \Phi_{b}=\Phi_{0} \Delta n+c \int_{0}^{t} \Delta T\left(\beta_{I I}-\beta_{I}\right) d t .
$$

Thus this particular loop is sensitive to the frequency and in this connection to the thermoelectric power difference between the junctions.

When the current exceeds the critical value potential difference $V=\beta \Delta T$ appears across the junction due to the thermoelectric effects. Since the thermoelectric power of the junction $I I$ differs from that at the junction $I$, the potential differences across the first and second junctions, $V_{I I}$ and $V_{I}$, respectively, will differ so that $\Delta V=V_{I I}-V_{I}=\left(\beta_{I I}-\beta_{I}\right) \Delta T$. The basic technique for the detection of extremely small voltage differences between two Josephson junctions by monitoring of magnetic flux change was firstly developed by Clarke ${ }^{12}$.

In the absence of any additional effects on the Cooper pairs, one would thus expect the net EMF in the loop containing the junctions to be $\left(\beta_{I I}-\beta_{I}\right) \Delta T \sim$ $10^{-11} V$ for the typical values of parameters $\left(\beta_{I I}-\beta_{I}\right) \sim 10^{-6} V / K$ and $\Delta T \sim$ $10^{-5} \mathrm{~K}$. For the loop of inductance $L$ the evolution of magnetic field is approximately governed by law $d \Phi_{b} / d t=-L d I_{l} / d t$. In this connection a nonvanishing value for $\Delta V$ would lead, according to (3) and (6), to a time varying current $I_{l}$ (from zero to the critical maximum value in the range of one number of the step $n): d I_{l} / c d t=$ $-1 / L \Delta V$, which will induce the above discussed flux $\Delta \Phi_{b}=c \int \Delta V d t$ through the loop in the linear regime.

There are at least two realistic processes which change the ideal behaviour magnetic field $\Phi_{b}$ described by equation (7): (i) the ohmic dissipation of current in the normal layer of the Josephson contacts and (ii) the dependence of maximum superconducting current, which can pass through the junctions without dissipation on the magnetic field.

A new experiment, in which the thermoelectric response creates saw-tooth flux (7) oscillating in time can be proposed. It will give a good opportunity to measure thermal effects in SNS structures and may be relevant for confirmation of some aspects of thermoelectric transport theory in the superconducting state. Let us roughly evaluate the possibilty of performing this experiment. Taking $\left(\beta_{I}-\beta_{I I}\right) \sim$ $10^{-8} \mathrm{~cm}^{1 / 2} \cdot \mathrm{g}^{1 / 2} \cdot \mathrm{s}^{-1} / K$ and $\Delta T \sim 10^{-5} K$ we obtain for $\Delta \Phi_{b}\left(\right.$ Gauss $\left.\cdot \mathrm{cm}^{2}\right) \sim$ $3 \times 10^{-3} \cdot \Delta t(s)$ in the range of one step $n$. Measuring such variations of magnetic flux is in the capacity of SQUID sensitivity and can be easily performed. The obtained result, in principle, may be used for construction of new type devices such as high sensitivity precise thermometers and thermocouples.

The main problem in observation in the flux (7) will be connected with generating thermal current $\vec{\jmath}_{s}=-\lambda \beta$ gradT comparable in magnitude with its critical 
value $\vec{\jmath}_{c}$, since the temperature difference across the junction is limited by a low temperature $T_{c}$ and small sizes of the junction.

The similar method of measurement has been used by Jain et al ${ }^{12}$ in null result experiment on confirmation of the strong equivalence principle for a charged massive particle. In their experiment the phase of Josephson contacts has been locked to an external microwave source.

It is interesting to mention that the predicted mechanism for production of magnetic field and current changing with time can be of crucial importance in astrophysics as an additional way (to the existed ones ${ }^{13}$ ) for generation of electromagnetic radiation from pulsars. According to the recent theoretical models ${ }^{14}$, a neutron star is the relativistic compact object consisting of the conducting crust and superfluid core. In the inner crust of the neutron star the superfluid coexists with a crystal lattice and in its core, at densities above $2 \times 10^{14} \mathrm{gm} / \mathrm{cm}^{3}$ there is a homogeneous mixture of superfluid neutrons and superconducting protons.

Important fact is that the thermoelectric power $\beta$ is the function of temperature as $T^{3 / 2}$ and in this connection can reach large numbers since superconductivity in the stars takes place at the temperatures $10^{6} \div 10^{7} \mathrm{~K}$. So if one accepts that the $S N S$ structures are realized in the intermediate boundary between conducting crust and superconducting core inside the neutron star then the strong heat fluxes in these $S N S$ junctions can lead to the generation of time-varying magnetic field (i.e. electromagnetic radiation) due to the thermoelectric effect described by the basic formula ( 7$)$.

\section{Acknowledgments}

The author acknowledges the financial support and hospitality at the Abdus Salam International Centre for Theoretical Physics, Trieste, where the main part of the work was done.

\section{References}

1. J. Clarke and S.M. Freake, Phys. Rev. Lett. 29, 588 (1972).

2. A.G. Aronov and Yu.M. Galperin, JETP Lett. 19, 165 (1974).

3. G.I. Panaitov, V.V. Ryazanov, A.V. Ustinov and V.V. Schmidt, Phys. Lett.A 100, 301 (1984).

4. V.V. Shmidt, JETP Lett. 33, 98 (1981).

5. V.V. Ryazanov and V.V. Schmidt, Solid State Comm. 40, 1055 (1981).

6. B.D. Josephson, Phys. Lett. 1, 251 (1962).

7. V.L. Ginzburg and G.F. Zharkov, Sov. Phys. Uspekhi 21, 381 (1978).

8. D.J. Van Harlingen, Physica 1098110B, 1710 (1982).

9. R.P. Huebener, Supercond. Sci. Technol. 8, 189 (1995).

10. Z.D. Wang, Q. Wang and P.C.W. Fung, Supercond. Sci. Tecnol. 9, 333 (1996).

11. J. Clarke, Phys. Rev. Lett. 21, 1566 (1968).

12. A.K. Jain, J. Lukens J and J.S. Tsai, Phys. Rev. Lett. 58, 1165 (1987).

13. F.C. Michel, Theory of Neutron Star Magnetospheres (Univ. Chicago Press, 
November 29, 2018 1:21 WSPC/INSTRUCTION FILE MPLB

On a Possibility to Measure Thermoelectric Power in SNS Structures

1991).

14. S.L. Shapiro and S.A. Teukolsky, Black Holes, White Dwarfs, and Neutron Stars (New York: Wiley, 1983). 